\title{
COMPOSTOS FENÓLICOS TOTAIS E ATIVIDADE ANTIOXIDANTE DA FARINHA DO RESIDUO DA UVA
}

\section{TOTAL PHENOLIC COMPOUNDS AND ANTIOXIDANT ACTIVITY OF GRAPE RESIDUE FLOUR}

\author{
Stefani Rafaeli Zopellaro, Sabrine Zambiazi da Silva ${ }^{2}$, Frederico Rodrigues Lovato³ \\ ${ }^{1}$ Nutricionista Centro Universitário Fundação Assis Gurgacz (FAG), ${ }^{2}$ Nutricionista. Mestre em \\ Engenharia Agrícola, professora do Centro Universitário (FAG) , ${ }^{3}$ Engenheiro Químico - FUNDETEC, \\ Cascavel - PR \\ *Autor correspondente: sazambiazi@yahoo.com.br, https://orcid.org/0000-0001-6931-3601
}

\begin{abstract}
RESUMO
A uva é fonte de compostos fenólicos, com vários efeitos benéficos ao organismo, como, ação antioxidante, anti-inflamatória, antiplaquetária e antialergênica. A utilização da uva na indústria alimentícia gera muito resíduo que normalmente, não é aproveitado como deveria, evitando a agregação de valor a um produto com grande potencial de utilização. Esse resíduo traz problemas econômicos e ambientais. Logo, o presente trabalho foi desenvolvido visando a utilização desse resíduo para produção de farinha e posterior análise dos compostos fenólicos totais e da atividade antioxidante. A produção da farinha se deu através das etapas de seleção da matéria prima, higienização das uvas, desengace, despolpamento, secagem em estufa a 55 ${ }^{\circ} \mathrm{C}$, trituração e moagem. Após a confecção, foi armazenada e levada para análises. A análise de antioxidantes foi realizada por espectrometria utilizando o reagente ABTS (ácido sulfônico), os resultados encontrados foram de 127,07 $\mu \mathrm{mol}$ de Trolox / g, o teor de compostos fenólicos totais foi de $8,13 \mathrm{mg} E A G / \mathrm{g}$. Quando os resultados obtidos são comparados a literatura, conclui-se que a farinha do resíduo da uva é fonte de compostos fenólicos, possuindo um bom potencial antioxidante, tornando-se uma alternativa para diminuir perdas econômicas dentro de uma empresa e consequentemente reduzir o impacto ambiental.
\end{abstract}

Palavras-chave: Uva; Compostos Fenólicos; Antioxidante.

Órgãos Financiadores: Fundação para o Desenvolvimento Científico e TecnológicoFUNDETEC.

\begin{abstract}
The grape is a source of phenolic compounds, with various beneficial effects to the body, such as antioxidant, anti-inflammatory, antiplatelet and anti-allergenic action. The use of grapes in the food industry generates a lot of waste that is normally not used as it should, avoiding the addition of value to a product with great potential for use. This waste brings economic and environmental problems. Therefore, the present work was developed aiming the use of this residue for flour production and subsequent analysis of total phenolic compounds and antioxidant activity. The production of the flour occurred through the stages of selection of the raw material, cleaning of the grapes, destemming, pulping, oven drying at $55^{\circ} \mathrm{C}$, and grinding. After the preparation, it was stored and taken for analysis. Antioxidant analysis was performed by spectrophotometry using the ABTS reagent (sulfonic acid), the results found were $127.07 \mu \mathrm{mol}$ Trolox / g, the total phenolic compounds content was $8.13 \mathrm{mg}$ EAG / g. When the obtained results are compared to the literature, it is concluded that the flour
\end{abstract}


of the residue of the grape is a source of phenolic compounds, possessing a good antioxidant potential, becoming an alternative to reduce economic losses within a company and consequently reduce the environmental impact.

Keywords: grape; phenolic compounds; antioxidant.

Funding Agencies: Foundation for Scientific and Technological DevelopmentFUNDETEC.

\section{INTRODUÇÃO}

O Brasil cultiva basicamente duas espécies de uva, a Vitis vinífera, destinada à elaboração de vinhos e outros produtos finos, pois possuem um custo elevado de produção e a Vitislabrusca, que corresponde com cerca de $80 \%$ da produção e por ter um custo menor é destinada para produção de vinhos de mesa, sucos e derivados e para o consumo in natura (CAMARGO, NACHTIGAL, 2007; SAUTTER, 2003).

A utilização da uva na indústria alimentícia acaba gerando muito resíduo, o qual normalmente não é aproveitado como deveria, evitando, assim, a agregação de valor a um produto com grande potencial de utilização. Na indústria vitivinícola, os principais resíduos agroindustriais são separados durante as etapas de esmagamento e prensagem das uvas, dos quais somente pequenas quantidades desses resíduos são valorizadas ou aproveitadas (MONRAD et al., 2010). Estima-se que 73 milhões de toneladas de uvas, principalmente, cultivadas como Vitisvinifera, são produzidas mundialmente (EMBRAPA, 2015), das quais o bagaço de uva representa aproximadamente $20 \%$ do volume total (ROCKENBACH et al., 2011).

"Resíduo é todo material descartado, individual ou coletivamente, pela ação humana, animal ou por fenômenos naturais, que seja nocivo à saúde, ao meio ambiente e, ao bem-estar da população" (FERRARI, 2010, p.4). Os resíduos sólidos da uva industrializada que podem ter interesse econômico são o bagaço, sementes, engaço, borras, grainhas, folhetos, sarro, além do material filtrado dos líquidos, dentre outros (FERRARI, 2010). O reaproveitamento desses resíduos pode contribuir para reduzir impactos ambientais e perdas econômicas, além de representar avanço na manutenção do equilíbrio do meio ambiente.

Dentre as várias alternativas existentes de incorporação dos resíduos em formulações alimentícias visando evitar o descarte inapropriado e o desperdício, destaca-se o aproveitamento para a produção de farinhas (PELISSARI et al., 2012; AZIZ et al., 2012.). A farinha do resíduo da uva pode ser utilizada para elaboração de biscoitos, pães, barras de cereais, massas caseiras, vitaminas, sucos, agregando valor nutricional ao produto (ARAUJO, 2010).

A uva é composta basicamente de açúcares, ácidos, pectinas, gomas, compostos aromáticos e compostos fenólicos. Durante a maturação, há uma evolução de alguns desses constituintes, dentre eles: açúcares, ácidos, compostos fenólicos, vitaminas, minerais, ocorrendo então crescimento da baga da uva, acumulação de açúcares, formação de taninos, diminuição de ácidos e consequentemente formação de aromas (PEIXOTO, 2000).

Vários efeitos benéficos à saúde têm sido atribuídos aos compostos fenólicos presentes nas frutas, vegetais, chás e vinhos. As uvas são consideradas uma das maiores fontes de compostos fenólicos quando comparadas a outras frutas e vegetais (ABE et al., 2007; VEDANA, 2008).

Pesquisas mostram que os compostos fenólicos estão associados à proteção contra doenças degenerativas, como doenças cardíacas, diabetes e câncer, isso pode ser justificado pelo seu poder antioxidante que age combatendo os radicais livres, os quais atacam biomoléculas, tais como lipídeos, proteínas e até mesmo DNA (GIADA; 
MANCINI FILHO, 2006). Além da capacidade antioxidante, os flavonoides ainda possuem ações anti-inflamatória, antiplaquetária e antialergênica (DEGÁSPARI; WASZCZYNSKYJ, 2004).

Cascas e sementes vêm sendo utilizadas para obtenção de ingredientes funcionais, tais como antioxidantes naturais e suplementos alimentares (MONRAD et al., 2010). As cascas representam, em média, $82 \%$ do peso seco do bagaço, contendo uma grande quantidade de compostos fenólicos, incluindo antocianinas, ácidos hidroxicinâmicos, catequinas e flavonóis (ROCKENBACH et al., 2011). Além disso, apresentam teores significativos de fibra alimentar (65-80\%), apresentando alto valor nutritivo (LLOBERA; CAÑELLAS, 2008). Com isso, o presente estudo teve como objetivo produção da farinha do resíduo da uva, bem como, analise dos compostos fenólicos totais e atividade antioxidante, visto que esse resíduo possui grandes propriedades nutricionais e sua utilização resulta em bom impacto ambiental e econômico.

\section{METODOLOGIA}

\subsection{MATERIA PRIMA E OBTENÇÃO DA FARINHA}

A uva foi cedida pela Fundação para o Desenvolvimento Cientifico e Tecnológico (FUNDETEC), localizada na cidade de Cascavel- PR, em que foi desenvolvida a farinha e as análises físico-químicas. A análise de compostos fenólicos totais e atividade antioxidante foram realizadas na Universidade Tecnológica Federal do Paraná (UTFPR), localizada na cidade de Toledo-PR.

Primeiramente, foi realizada a recepção da matéria-prima em boa qualidade, seguido da higienização da uva que foi enxaguada em água corrente para a remoção de sujidades e em seguida deixada submersa em solução de água clorada a 200 ppm por 15 minutos e foi novamente enxaguada em água corrente. Após a etapa de limpeza, é realizada o desengace, que consiste na retirada do cabinho da uva conhecido como engaço, que apresenta sabor amargo e adstringente ao produto. Em sequência, a uva foi levada para a despolpadeira, equipamento utilizado para separar a polpa da fruta do material fibroso, sementes, cascas, gerando o bagaço (resíduo) da uva. O bagaço foi embalado em sacos plásticos e armazenado em freezer a -20 ${ }^{\circ} \mathrm{C}$ para preservação das suas características, até a utilização. Posteriormente se retirou as embalagens para descongelamento durante 12horas.

A partir da seleção do resíduo, o mesmo foi submetido à secagem em estufa com circulação de ar da marca Nova Ética à temperatura de $55^{\circ} \mathrm{C}$ por 48 horas (2 dias). Após a retirada da estufa e posterior resfriamento do bagaço seco, triturou-se em processador de alimentos, obtendo-se a farinha. A ainda foi reprocessada em um moinho do tipo willey da marca Tecnal TE-650 em peneira média (16 a 24 mesh) para padronização do tamanho das partículas (Figura 1).

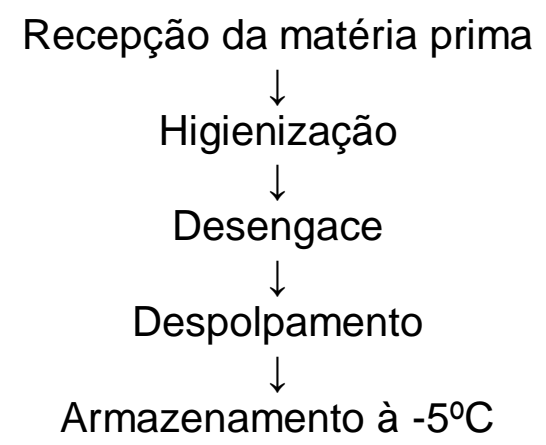

FAG Journal of Health - ISSN 2674-550X, 2019, v.1, n.2, p. 156 


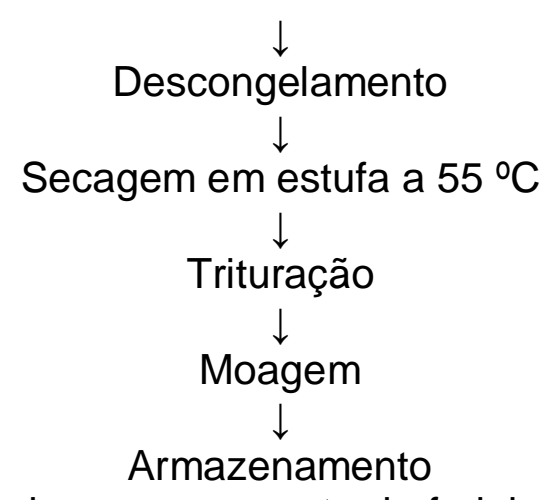

Figura 1. Fluxograma geral do processamento da farinha do resíduo da uva

Foi acondicionado em embalagem de polipropileno transparente com fechamento off-set e armazenado em local fresco e seco.

\subsection{ANALISE FÍSICO-QUÍMICA E DE ATIVIDADE ANTIOXIDANTE}

A análise físico-química da farinha foi realizada no laboratório da FUNDETEC, em triplicata, seguindo as metodologias descritas em IAL (2008). O teor de cinzas foi analisado após a incineração das amostras em mufla SP-1200 a $550^{\circ} \mathrm{C}$. A umidade foi determinada em secagem direta em estufa marca $4023 \mathrm{D}$ a $105^{\circ} \mathrm{C}$ até obter peso constante. O teor total de proteínas foi analisado pelo método de kjeldahl, o fator de conversão utilizado foi de 6,25 e o teor de lipídios deu-se em um extrator de soxhlet INV-06 (Inova) com o reagente éter de petróleo.

Para análise dos compostos fenólicos totais foi utilizada a metodologia FolinCiocalteau, descrita por Singleton e Rossi (1965). A análise é realizada através de espectrofotometria, a absorbância foi medida utilizando $760 \mathrm{~nm}$, os resultados dos compostos fenólicos totais foram expressos em equivalente de ácido gálico (mg EAG por 100,0 g de extrato), tendo como base a equação da reta obtida para a curva de calibração de ácido gálico previamente preparada e lida.

Para análise da atividade antioxidante foi utilizado o método ABTS (Ácido sulfônico) e foi realizado como descrito Boroski et al 2015. A análise consiste em um método de espectrofotometria e os resultados foram expressos em $\mu \mathrm{mol}$ de Trolox / g.

\subsection{Analise estatística}

Os dados da análise dos compostos fenólicos totais foram analisados estatisticamente através do Software Microssoft Excel.

\section{RESULTADOS E DISCUSSÕES}

\subsection{COMPOSIÇÃO QUIMICA}

Os resultados obtidos em relação à composição química da farinha são exibidos na Tabela 1.

O conteúdo proteico encontrado $(5,64 \mathrm{~g} / 100 \mathrm{~g})$ é inferior aos valores visto por Sousa et al. (2014), 8,49 g/100g e por Bender et al., (2016), 6,78 g/100 g. Bender et al., (2016) também encontrou valores inferiores de lipídeos $5,13 \mathrm{~g} / 100 \mathrm{~g}$.

Bampi et al (2010) avaliaram a composição centesimal de uma farinha feita a partir da Uva da variedade Uva-do-Japão e encontraram valores semelhantes de proteína $(5,73 \mathrm{~g} / 100 \mathrm{~g})$, teores menores de cinzas $(4,48 \mathrm{~g} / 100 \mathrm{~g})$ e lipídeos $(1,82 \mathrm{~g} /$ 
$100 \mathrm{~g})$ e quantidades superiores de umidade $(19,08 \mathrm{~g} / 100 \mathrm{~g})$. $O$ teor de umidade achado (8,43 g/100 g) está de acordo com os critérios exigidos pela RDC 263/2005 que estipula um teor máximo de umidade de $15 \%$ para farinhas obtidas de frutos e sementes (BRASIL, 2005).

Tabela 01. Analise da composição química da farinha do resíduo da uva

\begin{tabular}{ll}
\hline ANÁLISE & QUANTIDADE EM 100 GRAMAS \\
\hline Cinzas $(\mathrm{g})$ & $5,61 \pm 0,34$ \\
Umidade $(\mathrm{g})$ & $8,43 \pm 0,21$ \\
Proteínas $(\mathrm{g})$ & $5,64 \pm 0,40$ \\
Lipídeos $(\mathrm{g})$ & $6,20 \pm 0,05$ \\
\hline
\end{tabular}

Segundo o Ibravin (2010), as diferenças encontradas em relação a outros estudos, na composição química da farinha podem ocorrer devido fatores como, o estágio de maturação, variedade e época do ano e ainda em função de condições agroclimáticas e de atividades enológicas da região do vinhedo, como sistema de condução da vinha, irrigação, emprego de adubação e estado sanitário das uvas no momento da colheita.

\subsection{ATIVIDADE ANTIOXIDANTE}

Em relação a determinação da atividade antioxidante da farinha da uva foi realizada as análises dos compostos fenólicos totais e ABTS (Tabela 3).

TABELA 3. Resultados das análises de determinação da atividade antioxidante da farinha

\begin{tabular}{ll}
\hline Analise & Resultado \\
\hline ABTS $\mu$ mol de Trolox / g & $127,07 \pm 8,33$ \\
Fenólicos totais mg EAG/g & $8,13 \pm 0,91$ \\
\hline
\end{tabular}

Fonte: autor, 2017.

Com base na tabela 3, pode-se observar resultados inferiores de fenólicos totais quando comparados a estudos similares do autor Bender et al., que encontraram 2,78 mg EAG/ g (2016). No entanto, a pesquisa discutida foi utilizada somente as cascas para produção da farinha o qual pode resultar em alterações e diferenças dos resultados do estudo em epígrafe. Ainda, pode-se destacar que as sementes da uva são fontes importante de fenólicos totais. Os autores Yemis, Bakkalbasi e Artik (2008), avaliaram as sementes de sete variedades de uvas vermelhas cultivadas na Turquia, encontraram valores médios para fenóis totais de $50,41 \mathrm{mg} \mathrm{EAG} / \mathrm{g}$.

Os resultados de fenólicos totais do presente estudo são superiores quando comparados a casca da uva in natura, como no estudo de Soares et al., (2008) que avaliou o conteúdo de fenólicos em cascas de uva de duas variedades, a uva Isabel e Niágara e encontrou valores de 196,83 e 183,04 mg EAG/100 g de casca respectivamente. Por esse meio, o estudo de Rockenbach et al., (2011), que avaliaram a atividade antioxidante no bagaço da uva em quatro variedades de uva, total médio de compostos fenólicos totais encontrados foi de 61,62 mg EAG/100 g respectivamente. É importante ressaltar que os estudos nos estudos citados, os valores de fenólicos totais foram expressos em $\mathrm{mg} E A G / 100 \mathrm{~g}$, quando se faz a 
conversão dos resultados do presente trabalho para essa unidade de medida encontra-se $813,00 \mathrm{mg}$ EAG/100 g, valor superior ao dos autores.

Sánchez-Alonso, Jiménez-Escrig, Saura-Calixto e Borderías (2008) analisaram a quantidade total de polifenóis e atividade antioxidante pelo método ABTS contidos em fibra dietética extraída do bagaço de uva da variedade Airén, cultivada na Espanha. O resultado obtido foi de $78,5 \mathrm{mg}$ EAG/g para fenóis totais e $284 \mu \mathrm{Mol}$ TEAC/g para atividade antioxidante.

Valores semelhantes foram encontrados na pesquisa de Pérez-Jiménez et al., (2008) ao que se refere ao poder antioxidante da farinha pelo método ABTS. Os valores encontrados pelo autor foram de 124,4 $\mu \mathrm{M}$ de TEAC / g para uvas vermelhas produzidas em Manzanares, Espanha. Colaborando com a pesquisa, Rockenbach et al., (2011), quando avaliaram a atividade antioxidante no bagaço quatro variedades de uva por ABTS encontrou valores médios de $338 \mu$ MolTrolox/g.

Rockenbach et al., (2007) avaliaram o conteúdo total de polifenóis e a capacidade antioxidante de bagaços de uva das variedades Pinotnoir e Regente, inferindo que os extratos de bagaço de uva demonstram excelente potencial antioxidante atuando como inibidores de radicais livres. Resultados semelhantes foram encontrados por Campos et al., (2008) ao analisarem a atividade antioxidante do resíduo de vinificação produzido com uvas da variedade Cabernet sauvingnon.

Em relação ao resíduo, a Embrapa (2014), originou um insumo proveniente do bagaço da uva com alto teor de compostos funcionais que foi indicada para utilização em forma aquosa ou em pó pelas indústrias alimentícia, farmacêutica e cosmética. Os extratos concentrados obtidos a partir dos bagaços de uva apresentaram atividade antioxidante $50 \%$ maior que a polpa do açaí. O bagaço contém outro coproduto de alto valor agregado: a semente, que gera óleo vegetal. Por essa razão, o projeto também definiu condições para a extração do óleo de sementes de uva, a partir de resíduos do processamento por processo físico, ou seja, não utilizando solventes químicos derivados do petróleo. Os óleos obtidos a partir de resíduos da agroindústria apresentam, em geral, elevados índices de acidez verificados durante 0 processamento pós-colheita do fruto.

É importante destacar que vários fatores podem interferir no teor de fenólicos totais e na capacidade antioxidante, como as características agroclimáticas, espécie e estágio da maturação (IBRAVIN, 2010; PERESTRELO et al.,2012). Outro fator relevante é a temperatura utilizada no processamento visto que as antocianinas são rapidamente destruídas pelo aquecimento durante 0 processamento e armazenamento de alimentos. Estudos demonstraram relação logarítmica entre a destruição das antocianinas e o aumento aritmético da temperatura, portanto processos utilizando baixo tempo e alta temperatura tem sido recomendada para melhor retenção desse composto (MALACRIDA, MOTTA, 2006).

Shirahigue (2008) avaliou o emprego do extrato do bagaço de uva das variedades Niágara e Isabel, proveniente do processo de vinificação de cantinas do município de Videira - SC. O extrato obtido foi caracterizado quimicamente e aplicado em carne de frango processada, para avaliar o efeito desse composto sob a estabilidade oxidativa e a qualidade do produto cárneo em questão. O emprego do extrato de bagaço de uva foi eficiente para manter a estabilidade lipídica da carne de frango, apresentando resultados compatíveis com aqueles exibidos pelo antioxidante sintético BHT.

Mildner - Szkudlarz et al., (2013) avaliaram a incorporação do bagaço de uva em diferentes níveis (10, 20 e 30\%) nas formulações de biscoitos com a finalidade de avaliar a influência nas propriedades nutracêuticas, físicas e sensoriais. Concluíram 
que a incorporação aumentou gradualmente os potenciais antioxidantes e o teor de fibras e a dureza dos biscoitos.

O conhecimento sobre o potencial nutricional e antioxidante das diferentes partes da fruta (polpa, casca, caroço ou semente) contribui para o aumento da viabilidade de utilização das partes descartadas como insumo em formulações alimentícias, bem como agregar valor ao produto possibilitando a produção de novas formulações abrangendo potenciais consumidores que se preocupam com a manutenção da saúde, além de ser imprescindível para aumentar a eficiência do agronegócio e, consequentemente, reduzir o impacto ambiental. (ABDENNACER et al., 2015).

\section{CONSIDERAÇÕES FINAIS}

Por meio do trabalho desenvolvido, é possível constatar através dos resultados que a farinha feita a partir do resíduo da uva tem um potencial antioxidante e é fonte de compostos fenólicos. Esse achado torna relevante estudos abrangendo a avaliação da estabilidade desses compostos, após o processamento, quando utilizado para fabricação de produtos alimentícios, como biscoitos, pães, barras de cereais, massas caseiras, vitaminas e sucos, sendo uma forma de agregar valor nutritivo aos mesmos.

Além do mais, a utilização dos resíduos agroindustriais é um meio para se diminuir as perdas econômicas dentro de uma empresa e consequentemente reduzir o impacto ambiental.

\section{REFERÊNCIAS BIBLIOGRÁFICAS}

ABDENNACER, B. et al. Determination of phytochemicals andantioxidant activity of methanolextracts obtained from the fruitand leaves of Tunisian Lyciumintricatum Boiss. FoodChemistry, v. 174, p. 577-584, [S.I.], 2015.

ABE, L. T. et al. Compostos fenólicos e capacidade antioxidante de cultivares de uvas vitis labrusca I. e vitis vinifera I. Ciênc. tecnol. aliment, Campinas, v. 27, n. 2, p. 394400, abr./jun. 2007.

ARAÚJO, J. Como fazer farinha de uva. 2010, disponível em: <\&lt;http://blog.jarioaraujo.com/2010/nutricao/143/como-fazer-farinha-de-uva/\&gt>. Acesso em: 03 abr. 2017.

AZIZ, N. A. A.; WONG, L. M.; BHAT, R. ; CHENG, L. H. Evaluation of processed green and ripe mango peel and pulp flours (Mangifera indica var. Chokanan) in terms of chemical composition, antioxidante compounds and functional Properties. Journal of Science Food of Agriculture, London, v.92, n.92, p.557-563, 2012.

BAMPI, M. et al. Composição centesimal do fruto, extrato concentrado e da farinha da uva-do-japão. Cienc. Rural, Santa Maria, v. 40, n. 11, p. 2361-2367, Nov. 2010.

BENDER A. B. B. et al. Obtenção e caracterização de farinha de casca de uva e sua utilização em snack extrusado. 2011. Dissertação (Mestrado em Ciência e Tecnologia de Alimentos) - Escola Superior de Agricultura "Luiz de Queiroz", Universidade de São Paulo, Piracicaba. 
BOROSKI M. et al. Antioxidantes: princípios e métodos analíticos.1 ed., Curitiba, 2015.

BRASIL. Ministério da Saúde. Agência Nacional de Vigilância Sanitária. Resolução nㅇ 263, de 22 de setembro de 2005. Dispõe sobre o regulamento técnico para produtos de cereais, amidos, farinhas e farelos. Diário Oficial [da] República Federativa do Brasil, Brasília, DF, 22 set. 2005. Seção 1. Disponível em: < http://portal.anvisa.gov.br/wps/wcm/connect/b683960047457>. Acesso em 20 mai. 2017.

CAMARGO, U. A.; NACHTIGAL, J. C. Recomendações para produção de videiras em sistemas de base ecológica. Embrapa Uva e Vinho, 2007. Disponível em:< http://www.cnpuv.embrapa.br/publica/documentos/doc065.pdf>. Acesso em: 03 abr. 2017.

CAMPOS, L. M. A. S. et al. Experimental data and modeling the supercritical fluid extraction of marigold (Calendulaof.cinalis) oleoresin. Journal of Supercritical Fluids. v.34, n.2, p.163-170, 2005.

DEGÁSPARI, C. H.; WASZCZYNSKY, N. Propriedades antioxidantes de compostos fenólicos antioxidants properties of phenolic compounds. Visão acadêmica, Curitiba, v. 5, n. 1, p. 33-40, jan./jun. 2004.

EMPRESA BRASILEIRA DE PESQUISA AGROPECUÁRIA - EMBRAPA. Bagaço de uva vira alimentos funcionais. Brasília: Embrapa, 2014. Disponível em:<https://www.embrapa.br/busca-de-noticias/-/noticia/2235712/bagaco-de-uvavira-alimentos-funcionais>. Acesso em: 03 abr. 2017.

EMPRESA BRASILEIRA DE PESQUISA AGROPECUÁRIA - EMBRAPA. Nota técnica: um balanço da Vitivinicultura Mundial em 2014. Brasília: Embrapa, 2015. Disponível em:<https://www. embrapa.br/busca-de-noticias/-/noticia/4007952/notatecnica-- --um-balanco-da-vitivinicultura-mundial-em-2014>. Acesso em: 03 abr. 2017.

FERRARI, V. A. Sustentabilidade da vitivinicultura através de seus próprios resíduos. 2010. Trabalho de Conclusão de Curso (Bacharelado em Ciências Econômicas) - (Universidade de Caxias do Sul, Caxias do Sul, 2010.

FERREIRA L. F. D. Obtenção e caracterização de farinha de bagaço de uva e sua utilização em cereais matinais expandidos. 2010. 135 f. Tese (Doutorado em Ciência e Tecnologia de Alimentos) - Universidade Federal de Viçosa, Viçosa, 2010.

GIADA, M. L. R.; MANCINI FILHO J. Importância dos compostos fenólicos da dieta na promoção da saúde humana. Ci. biol. saúde, Ponta grossa , v. 12, n. 4, p. 7-15, dez. 2006.

IAL (INSTITUTO ADOLFO LUTZ). Normas Analíticas do Instituto Adolfo Lutz: Métodos físico-químicos para análise de alimentos. Instituto Adolfo Lutz. São Paulo, 2008. 
INSTITUTO BRASILEIRO DE VINHOS - IBRAVIN. A vitivinicultura brasileira. Bento Gonçalves: IBRAVIN, 2010. Disponível em:< http://www.ibravin.org.br/> Acesso em: 15 jul. 2017.

Isolation and characterization of the flour and starch of plantain bananas (Musa paradisiaca). Starch/Stazrke. v. 64, n. 5, p. 382-391, 2012.

LLOBERA, A.; CAÑELLAS, J. Antioxidant activity and dietary fibre of Prensal Blanc White grape (Vitis vinifera) by-products. International Journal of Food Science \& Technology Oxford. v. 43, n. 11, p. 1953-1959, 2008.

MALACRIDA, R. A., MOTTA, S. Antocianinas em Suco de Uva: Composição e Estabilidade. Boletim do Centro de Pesquisa de Processamento de Alimentos, Curitiba, v. 26, p. 59-82, 2006.

MILDNER- SZUDLARZ, S. et al. White grapepomace as a source of dietary fibre and polyphenols and its effect on physical and nutraceutical characteristics of wheat biscuits. J SciFood Agric. v. 93, p. 389 - 395, 2013

MONRAD, J. K.; HOWARD, L. R.; KING, J. W.; SRINIVAS, K.; MAUROMOUSTAKOS, A. Subcritical solvente xtraction of anthocyanin from dried red grape pomace. Journal of Agricultural and Food Chemistry, Washington, v. 58, n. 5, p. 2862-2868, 2010.

PEIXOTO, C. Enologia e outras bebidas. Disponível em:<http://opac.iefp.pt:8080/images/winlibimg. aspx?skey=\&doc=69677\&img=705> . Acessoem: 03 abr. 2017.

PELISSARI, F. M.; MAHECHA, M. M. A.; SOBRAL, P. J. A.; MENEGALLI, F. C. Isolation and characterization of the flour and starch of plantain bananas (Musa paradisiaca).Starch/Stazrke. v. 64, n. 5, p. 382-391, 2012.

PERESTRELO, R. et al. Phenolic profile of sercial and tinta negra Vitis vinifera L. grape skins by HPLC-DAD-ESI-MSn Novel phenolic compounds in Vitis vinifera L. grape. Food Chemistry, Kidlington, v. 135, p. 94-104, 2012.

PÉREZ-JIMÉNEZ J. et al. Updated methodology to determine antioxidant capacity in plant foods, oils and beverages: Extraction, measurement and expression of results. Food Research International, v. 41 (2008), p. 274-285, 2008.

ROCKENBACH, I. I.; RODRIGUES, E.; GONZAGA, L. V.; CALIARI, V.; GENOVESE, M. I.; GONÇALVES, A. E. S. S.; FETT, R. Phenolic compounds contentandantioxidantactivity in pomace from selected red grapes (Vitisvinifera L. and Vitislabrusca L.) widely produced in Brazil. FoodChemistry, London, v. 127, n. 1, p. 174-179, 2011.

ROCKENBACH, I.I. et al. Influência do solvente no conteúdo total de polifenóis, antocianinas e atividade antioxidante de extratos de bagaço de uva (Vitisvinifera) variedades Tannat e Ancelota. Ciência e Tecnologia de Alimentos, Campinas, v.28, p.238-244, 2008. Disponível em:<http://www.scielo.br/scielo.php?pid=S010120612008000500036\&script=sci_arttext>. Acesso em: 15 mar. 2017 
SÁNCHEZ-ALONSO, A.; JIMÉNEZ-ESCRIG, F.; SAURA-CALIXTO, A. J. Borderías Antioxidant protection of White grape pomace on restructured fish products during frozens torage. LWT - Food Science and Technology, v. 41, p. 42-50, 2008.

SAUTTER, C. K. Avaliação da presença de resveratrol em suco de uva. Santa Maria, 2003, 135 f. Dissertação (Mestrado em Ciência e Tecnologia dos Alimentos) Programa de PósGraduação em Ciência e Tecnologia dos Alimentos, Universidade Federal de Santa Maria (UFSM), 2003.

SHIRAHIGUE, L. D.; Caracterização química de extratos de semente e casca de uva e seus efeitos antioxidantes sobre carne de frango processada e armazenada sob refrigeração. Piracicaba, 2008. Disponível em: < www.teses.usp.br/teses/disponiveis/11/11141/tde-07082008- 121725/.../ligianne.pdf> Acesso em: 15 mar. 2017.

SINGLETON, V.L.; ROSSI, J.A. "Colorimetryof Total PhenolicswithPhosphomolybdicPhosphotungsticAcidReagents," PhenolicsDetermination, vol.16, n. 3, p. 144-158, set 1965.

SOARES, S. E. Ácidos fenólicos como antioxidantes. Rev. Nutr., Campinas, v. 15, n. 1, p. 71-81, Jan. 2002 Disponivel em <http://www.scielo.br/scielo.php?script=sci_arttext\&pid=S141552732002000100008\& lng=en\&nrm=iso $>$. Acesso em 10 de mai. de 2017.

VEDANA, M. I. S. Efeito do processamento na atividade antioxidante da uva. 2008, 88 f. Dissertação (Mestrado em Tecnologia em Alimentos) Universidade Federal do Paraná (UFPR).

YEMIS, O.; BAKKALBASSI E.; ARTIK, N. Antioxidative activities of grape (Vitis vinifera) seed extracts obtained from different varieties grown in Turkey. International Journal of Food Science and Technology, v. 43, p. 154-159, (2008). 